\title{
Critical behavior of disordered systems with replica symmetry breaking
}

\author{
P. V. Prudnikov, V. V. Prudnikov \\ Omsk State University, \\ Omsk, 644077 Russia
}

\begin{abstract}
A field-theoretic description of the critical behavior of weakly disordered systems with a $p$-component order parameter is given. For systems of an arbitrary dimension in the range from three to four, a renormalization group analysis of the effective replica Hamiltonian of the model with an interaction potential without replica symmetry is given in the two-loop approximation. For the case of the onestep replica symmetry breaking, fixed points of the renormalization group equations are found using the Pade-Borel summing technique. For every value $p$, the threshold dimensions of the system that separate the regions of different types of the critical behavior are found by analyzing those fixed points. Specific features of the critical behavior determined by the replica symmetry breaking are described. The results are compared with those obtained by the $\varepsilon$-expansion and the scope of the method applicability is determined.
\end{abstract}

\section{Introduction}

When the renormalization group approach is applied to describe the critical behavior of disordered systems with quenched disorder, the method of replicas [1, 2, 3] is used to restore the translation symmetry of the effective Hamiltonian describing the interaction of fluctuations. However, in the studies [4, 5, 6], it was conjectured that the replica symmetry could be broken in systems with quenched disorder. In [4, 5, 6], the physical concept of the occurrence of numerous local energy minima in disordered systems with random transition temperature was used to give a renormalization group description of the $\phi^{4}$ model with the interaction potential characterized by the broken replica symmetry. For this purpose, the $\epsilon$-expansion technique was used in the lower order of the theory. For systems in which the number of components of the order parameter $p$ is less than four, it was discovered that the breaking of replica symmetry is the crucial factor in the critical behavior. It was 
shown that, for $p$ in the range from one through four, two modes of the system behavior are possible. The first one determines a nonuniversal critical behavior, which depends on seed values of the model parameters and, ultimately, on the concentration of impurities in the system. The second mode is characterized by the absence of a stable critical behavior, as also is the most interesting case of Ising $(p=1)$ systems. Even though the implications of these studies are very interesting, the results of a field-theoretic description of certain homogeneous and disordered systems in the two-loop and higher order approximations based on the asymptotic series summation techniques [7] showed that the stability analysis of various types of critical behavior that uses the first-order terms of the $\epsilon$-expansion can be considered only as a coarse estimate, especially for multivertex statistical models [8] For this reason, the results of investigation of the replica symmetry breaking (RSB) effects obtained in 4, 5, 6] require revaluation from the viewpoint of a more accurate approach.

To this end, we proposed in 9, 10, in the framework of the field-theoretic approach, a renormalization group description of the model of weakly disordered three- and twodimensional systems with the fourth-order interaction potential with respect to the order parameter fluctuations, which determines the replica symmetry breaking. An analysis of solutions to the renormalization group equations carried out in the two-loop approximation with the sequential application of the summation technique for Pade-Borel series showed that the critical behavior of three- and two-dimensional systems is stable with respect to the relative influence of the RSB effects, and the former scenario of the quenched disorder influence on the critical behavior is realized [11].

However, the scope of the results obtained in 4, 5] remains unclear. In particular, it is interesting to establish the threshold dimensions of the disordered system, $d_{c}(p)$, that separate the domain of influence of RSB effects from the critical behavior domains in which these effects are insignificant. It is also interesting to apply the renormalization group approach to analyze the behavior of systems with replica symmetry breaking in which no stable critical behavior exists and the strong coupling mode occurs (see [4, 5]). A theoretical analysis of this phenomenon is especially important from the viewpoint of the possible manifestation of RSB effects in strongly disordered systems and their observation in computer models of the critical behavior under the impurity concentration exceeding the impurity percolation threshold when extended impurity structures are formed in the system [12]. 
This paper is devoted to the consideration of the above-mentioned problems. For weakly disordered systems of an arbitrary dimension in the range from three to four, an analysis of the critical behavior of the model with an RSB potential is carried out based on the renormalization group approach in the two-loop approximation with the use of summation techniques. Our analysis does not rely on the $\varepsilon$-expansion technique.

\section{Definition of the model and the calculation proce- dure}

The model Ginsburg-Landau Hamiltonian, which describes the behavior of a $p$-component spin system with weakly quenched disorder near the critical point has the form

$$
H=\int d^{d} x\left\{\frac{1}{2} \sum_{i=1}^{p}\left[\nabla \phi_{i}(x)\right]^{2}+\frac{1}{2}[\tau-\delta \tau(x)] \sum_{i=1}^{p} \phi_{i}^{2}(x)+\frac{1}{4} g \sum_{i, j=1}^{p} \phi_{i}^{2}(x) \phi_{j}^{2}(x)\right\}
$$

where the random phase transition temperature has the Gaussian distribution $\delta \tau(x)$ with the variance $<<(\delta \tau(x))^{2}>>\sim u$, which is determined by a positive constant $u$ and is proportional to the concentration of the structure defects. The application of the conventional replica method (see, for example, [6]) makes it possible to average over the temperature fluctuations $\delta \tau(x)$ and reduce the problem of the statistical description of a weakly disordered system to the problem of the statistical description of a homogeneous system with the effective Hamiltonian

$$
H_{n}=\int d^{d} x\left\{\frac{1}{2} \sum_{i=1}^{p} \sum_{a=1}^{n}\left[\nabla \phi_{i}^{a}(x)\right]^{2}+\frac{1}{2} \tau \sum_{i=1}^{p} \sum_{a=1}^{n}\left[\phi_{i}^{a}(x)\right]^{2}+\frac{1}{4} \sum_{i, j=1}^{p} \sum_{a, b=1}^{n} g_{a b}\left[\phi_{i}^{a}(x)\right]^{2}\left[\phi_{j}^{b}(x)\right]^{2}\right\},
$$

Here, the index $a$ enumerates replicas (images) of the original homogeneous component in Hamiltonian (11); and the additional vertex $u$, which occurs in the interaction matrix $g_{a b}=g \delta_{a b}-u$, , specifies the effective interaction of fluctuations of the $(n \times p)$-component order parameter through ground state of the system with the configuration $\phi(x)=0$ ( at $T \geq T_{c}$ ), is performed at the scale of the correlation length, which turns to infinity the defect field. This statistical model is thermodynamically equivalent to the original disordered model in the limit $n \rightarrow 0$.

The subsequent renormalization group procedure, which statistically takes into account the contribution of long-wave fluctuations of the order parameter relative to the at the transition temperature $T_{c}$. This procedure makes it possible to analyze possible 
types of the critical behavior and conditions of their realization and calculate the critical indexes.

However, it was shown in [4, 5, 6] that a macroscopically large number of spatial regions with $\phi(x) \neq 0$ appears in the system due to fluctuations of the random transition temperature at $[\tau-\delta \tau(x)]<0$. These regions are separated from the ground state by potential barriers. To describe the statistical properties of systems with multiple local energy minima, the replica symmetry breaking formalism (suggested by Parisi) was used in 4, 5, 6] by analogy with spin glasses [9. According to the reasoning presented in [4, 5. 6], the statistical calculation of the contribution of nonperturbation degrees of freedom associated with the order parameter fluctuations relative to the configurations of the field $\phi(x)$ at the local energy minima results (when the replica procedure is applied for the weak disorder) in the appearance of additional interactions of the form in $\sum_{a, b} g_{a b} \phi_{a}^{2} \phi_{b}^{2}$ the effective replica Hamiltonian. Here, the final matrix $g_{a b}$ is no longer replica symmetric with $g_{a b}=g \delta_{a b}-u$, but rather has the RSB Parisi replica structure [13]. According to [4, 15, 6, 13, the matrix $g_{a b}$ with the RSB structure is parameterized (in the limit $n \rightarrow 0$ ) in terms of its diagonal elements $\tilde{g}$ and the off-diagonal function $g(x)$, which is defined on the interval $0<x<1: g_{a b} \rightarrow(\tilde{g}, g(x))$. Here, operations with the matrices $g_{a b}$ are defined by the rules

$g_{a b}^{k} \rightarrow\left(\tilde{g}^{k} ; g^{k}(x)\right), \quad\left(\hat{g}^{2}\right)_{a b}=\sum_{c=1}^{n} g_{a c} g_{c b} \rightarrow(\tilde{c} ; c(x)), \quad\left(\hat{g}^{3}\right)_{a b}=\sum_{c, d=1}^{n} g_{a c} g_{c d} g_{d b} \rightarrow(\tilde{d} ; d(x))$

where

$$
\begin{aligned}
\tilde{c} & =\tilde{g}^{2}-\int_{0}^{1} d x g^{2}(x), \quad c(x)=2\left[\tilde{g}-\int_{0}^{1} d y g(y)\right] g(x)-\int_{0}^{x} d y[g(x)-g(y)]^{2}, \\
\tilde{d} & =\tilde{c} \tilde{g}-\int_{0}^{1} d x c(x) g(x), \quad d(x)=\left[\tilde{g}-\int_{0}^{1} d y g(y)\right] c(x)+\left[\tilde{c}-\int_{0}^{1} d y c(y)\right] g(x)- \\
& -\int_{0}^{x} d y[g(x)-g(y)][c(x)-c(y)] .
\end{aligned}
$$

The replica symmetric situation corresponds to $g(x)=$ const, which is independent of $x$.

The renormalization group description of the model specified by the replica Hamiltonian (2) was carried out in the framework of the field-theoretic approach in the two-loop approximation for systems of an arbitrary dimension in the range from three to four. Possible types of critical behavior and their stability in the fluctuation domain are determined by the renormalization group equation for the coefficients of the matrix $g_{a b}$. They 
were determined by the conventional method based on the Feynman diagram technique for the vertex parts of the irreducible Grin functions and the renormalization procedure. For example, in the two-loop approximation, the two-point vertex function $\Gamma^{(2)}$, the fourpoint vertex functions $\Gamma_{a b}^{(4)}$, and the two-point function $\Gamma_{a a}^{(2,1)}$ with the insertion $\left(\phi_{i}^{a}\right)^{2}$ have the form

$$
\begin{aligned}
\left.\frac{\partial \Gamma^{(2)}}{\partial k^{2}}\right|_{k^{2}=0} & =1+4 f g_{a a}^{2}+2 p f \sum_{c=1}^{n} g_{a c} g_{c a} \\
\left.\Gamma_{a b}^{(4)}\right|_{k_{i}=0} & =g_{a b}-p \sum_{c=1}^{n} g_{a c} g_{c b}-4 g_{a a} g_{a b}-4 g_{a b}^{2}+(8+16 h) g_{a b}^{3}+(24+8 h) g_{a a}^{2} g_{a b}+ \\
& +48 h g_{a a} g_{a b}^{2}+4 g_{a a} g_{b b} g_{a b}+8 p h \sum_{c=1}^{n} g_{a c} g_{c b}^{2}+8 p h g_{a b} \sum_{c=1}^{n} g_{a c} g_{c b}+4 p h g_{a b} \sum_{c=1}^{n} g_{a c}^{2}+ \\
& +2 p \sum_{c=1}^{n} g_{a c} g_{c c} g_{c b}+4 p g_{a a} \sum_{c=1}^{n} g_{a c} g_{c b}+p^{2} \sum_{c, d=1}^{n} g_{a c} g_{c d} g_{d b}, \\
\left.\Gamma_{a a}^{(2,1)}\right|_{k_{i}=0} & 1-p \sum_{c=1}^{n} g_{c a}-2 g_{a a}+2 p g_{a a} \sum_{c=1}^{n} g_{c a}+(4+12 h) g_{a a}^{2}+ \\
& +6 p h \sum_{c=1}^{n} g_{c a}^{2}+p \sum_{c=1}^{n} g_{c c} g_{c a}+p^{2} \sum_{c, d=1}^{n} g_{d c} g_{c a},
\end{aligned}
$$

where the notation

$$
\begin{aligned}
& f(d)=-\left.\frac{1}{J^{2}} \frac{\partial}{\partial k^{2}} \int \frac{d^{d} k_{1} d^{d} k_{2}}{\left(k_{1}^{2}+1\right)\left(k_{2}^{2}+1\right)\left(\left(k_{1}+k_{2}+k\right)^{2}+1\right)}\right|_{k^{2}=0}, \\
& h(d)=\frac{1}{J^{2}} \int \frac{d^{d} k_{1} d^{d} k_{2}}{\left(k_{1}^{2}+1\right)^{2}\left(k_{2}^{2}+1\right)\left(\left(k_{1}+k_{2}\right)^{2}+1\right)}, \quad J=\int d^{d} k /\left(k^{2}+1\right)^{2},
\end{aligned}
$$

is used, and the redefinition $g_{a b} \rightarrow g_{a b} / J$ is carried out. The diagram representation of the corresponding contributions to $\Gamma^{(2)}, \Gamma_{a b}^{(4)}$ and $\Gamma_{a a}^{(2,1)}$ is shown in 2 .

However, the subsequent renormalization procedure for the vertex functions and the calculation of the $\beta$ and $\gamma$ functions, which determine the renormalization group transformations for the interaction constants, are difficult due to the complicated structure of relations (3) and (4) defining operations with matrices $g_{a b}$. The step-like structure of the function $g(x)$ established in [4, 5, 6] makes it possible to implement the renormalization procedure. In this paper, we restrict ourselves to the consideration of the one-step function $g(x)$ :

$$
g(x)=\left\{\begin{array}{l}
g_{0}, 0 \leq x<x_{0} \\
g_{1}, x_{0}<x \leq 1
\end{array}\right.
$$




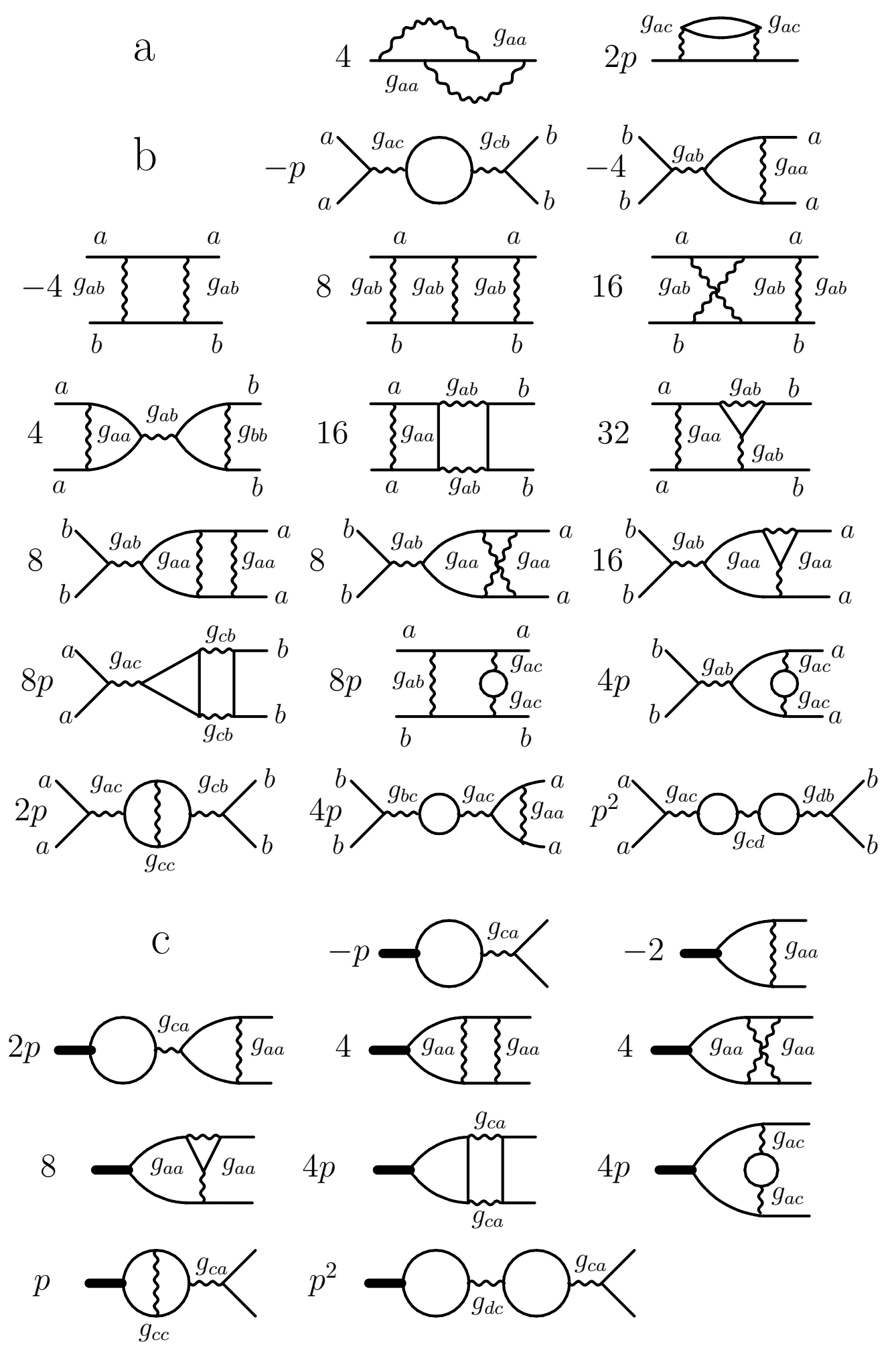

Figure 1: The diagram representation of contributions to the two-point $\Gamma^{(2)}$ (a), fourpoint $\Gamma_{a a}^{(2,1)}(\mathrm{b})$, and with the inclusion $\left(\phi_{i}^{a}\right)^{2}(\mathrm{c})$ vertex functions in the one- and two-loop approximations with the corresponding weighting coefficients. 
where the coordinate of the step $0 \leq x_{0} \leq 1$ is an arbitrary parameter that does not evolve under scale transformations and remains the same as in the seed function $g_{0}(x)$. As a result, the renormalization group transformations of the replica Hamiltonian with RSB are determined by the three parameters $\tilde{g}, g_{0}, g_{1}$.

The critical properties of the model can be revealed by analyzing the coefficients $\beta_{i}\left(\tilde{g}, g_{0}, g_{1}\right)(i=1,2,3), \gamma_{\phi}\left(\tilde{g}, g_{0}, g_{1}\right)$, and $\gamma_{\phi^{2}}\left(\tilde{g}, g_{0}, g_{1}\right)$ of the renormalization group Callan-Symanzik equation [14. We obtained the following expressions for the $\beta$ - and $\gamma$ functions in the two-loop approximation in the form of series in the renormalized parameters $\tilde{g}, g_{0}$ and $g_{1}$ :

$$
\begin{aligned}
\beta_{1} & =-\tilde{g}+(8+p) \tilde{g}^{2}-p x_{0} g_{0}^{2}-p\left(1-x_{0}\right) g_{1}^{2}+[(8 f-40 h+20) p \\
& +16 f-176 h+88] \tilde{g}^{3}+(24 h-8 f-12) x_{0} p \tilde{g} g_{0}^{2}+(24 h-8 f \\
& -12)\left(1-x_{0}\right) p \tilde{g} g_{1}^{2}-(16 h-8) x_{0} p g_{0}^{3}-(16 h-8)\left(1-x_{0}\right) p g_{1}^{3}, \\
\beta_{2} & =-g_{0}+(4+2 p) \tilde{g} g_{0}+\left(2 p x_{0}-4\right) g_{0}^{2}+2\left(1-x_{0}\right) p g_{0} g_{1} \\
& +[(8 f-48 h+28) p+16 f-48 h+24] \tilde{g}^{2} g_{0}-\left[\left((32 h-16) x_{0}\right.\right. \\
& +8-32 h) p+48-96 h] \tilde{g} g_{0}^{2}-(32 h-16)\left(1-x_{0}\right) p \tilde{g} g_{0} g_{1} \\
& +\left[(48 h-8 f-20) x_{0} p-32 h+16\right] g_{0}^{3}+(32 h-8)\left(1-x_{0}\right) p g_{0}^{2} g_{1} \\
& +(16 h-12-8 f)\left(1-x_{0}\right) p g_{0} g_{1}^{2}, \\
\beta_{3} & =-g_{1}+p x_{0} g_{0}^{2}-\left[p\left(x_{0}-2\right)+4\right] g_{1}^{2}+(4+2 p) \tilde{g} g_{1}+[(8 f-48 h \\
& +28) p+16 f-48 h+24] g_{1} \tilde{g}^{2}-(16 h-8) x_{0} p \tilde{g} g_{0}^{2}-\left[\left((8-16 h) x_{0}\right.\right. \\
& -8) p+48-96 h] u_{0} g_{1}^{2}+(16 h-8) x_{0} p g_{0}^{3}+(8 h-8 f-4) x_{0} p g_{1} g_{0}^{2} \\
& +\left[(8 f-24 h+12) x_{0} p+(48 h-8 f-20) p+16-32 h\right] g_{1}^{3}, \\
\gamma_{\phi} & =4(4-d) f(d)\left[(p+2) \tilde{g}^{2}-p x_{0} g_{0}{ }^{2}-p\left(1-x_{0}\right) g_{1}^{2}\right], \\
\gamma_{\phi^{2}} & =-(4-d)\left[(p+2) \tilde{g}+p x_{0} g_{0}+p\left(1-x_{0}\right) g_{1}-2(6 h-2 f-3)\left((p+2) \tilde{g}^{2}\right.\right. \\
& \left.\left.-p x_{0} g_{0}^{2}-p\left(1-x_{0}\right) g_{1}^{2}\right)\right] .
\end{aligned}
$$

In order to compare the results of this paper with those obtained in [4, [5, 6], we reversed, by analogy with [4, 5, 6], the signs of the off-diagonal elements in the matrix: $g_{a \neq b} \rightarrow-g_{a \neq b}$. As a result, $g_{0}$ and $g_{1}$ became positive definite. The integrals $f(d)$ and $h(d)$ were calculated numerically for $3 \leq d<4$.

It is well known that the series used in perturbation theory are asymptotic, and vertices of the interaction of the order parameter fluctuations in the fluctuation domain $\tau \rightarrow 0$ 
are sufficiently large to directly apply expressions (10). For this reason, in order to extract the physical information from those expressions, we use the generalized (for the three-parameter case) Pade-Borel method for summing asymptotic series. The direct and inverse Borel transformations have the form

$$
\begin{aligned}
& f\left(\tilde{g}, g_{0}, g_{1}\right)=\sum_{i, j, k} c_{i j k} \tilde{g}^{i} g_{0}^{j} g_{1}^{k}=\int_{0}^{\infty} e^{-t} F\left(\tilde{g} t, g_{0} t, g_{1} t\right) d t \\
& F\left(\tilde{g}, g_{0}, g_{1}\right)=\sum_{i, j, k} \frac{c_{i j k}}{(i+j+k) !} \tilde{g}^{i} g_{0}^{j} g_{1}^{k} .
\end{aligned}
$$

In order to find the analytic continuation of the Borel image of a function, we use the following series in the auxiliary variable $\theta$

$$
\tilde{F}\left(\tilde{g}, g_{0}, g_{1}, \theta\right)=\sum_{k=0}^{\infty} \theta^{k} \sum_{i=0}^{k} \sum_{j=0}^{k-i} \frac{c_{i, j, k-i-j}}{k !} \tilde{g}^{i} g_{0}^{j} g_{1}^{k-i-j}
$$

The Pade approximation $[\mathrm{L} / \mathrm{M}]$ is applied to this series at the point $\theta=1$. This technique was successfully used in [8] to describe the critical behavior of certain systems characterized by several interaction vertices of the order parameter fluctuations. The symmetry conservation property of a system when applying a Pade approximant in the variable . becomes essential in the description of multivertex models. In this paper, we used the $[2 / 1]$ approximant for the calculation of $\beta$-functions in the two-loop approximation.

\section{Calculation results}

It is well known that the nature of the critical behavior is determined by the existence of a stable fixed point satisfying the system of equations

$$
\beta_{i}\left(\tilde{g}^{*}, g_{0}^{*}, g_{1}^{*}\right)=0 \quad(i=1,2,3)
$$

By numerically solving system (13) for functions found by the Pade Borel summation technique (for $p=1,2,3$ ), three types of nontrivial fixed points were found in the physically interesting domain of parameters $\tilde{g}^{*}, g_{0}^{*}, g_{1}^{*} \geq 0$ (see Tables 1-3). The firsttype fixed point with $\tilde{g}^{*} \neq 0, g_{0}^{*}=g_{1}^{*}=0$ corresponds to the critical behavior of the homogeneous system; the second-type fixed point with $\tilde{g}^{*} \neq 0$ and $g_{0}^{*}=g_{1}^{*} \neq 0$ corresponds to the critical behavior of the disordered system with replica symmetry; and the thirdtype fixed point with $\tilde{g}^{*} \neq 0, g_{0}^{*}=0$ and $g_{1}^{*} \neq 0$ corresponds to the critical behavior of the disordered system with RSB. The values of and at the fixed point with RSB depend on the 
coordinate of the step $x_{0}$. Tables $1-3$ present the values of and for $0 \leq x_{0} \leq 1$ with the step $\Delta x_{0}=0,1$ The possibility to realize one or another type of the critical behavior for each $p$ depends on the stability of the corresponding fixed point. The stability criterion of a fixed point reduces to a condition that the eigenvalues $\lambda_{i}$ of the matrix

$$
B_{i, j}=\frac{\partial \beta_{i}\left(\tilde{g}^{*}, g_{0}^{*}, g_{1}^{*}\right)}{\partial g_{j}}
$$

belong to the complex right half-plane.

An analysis of $\lambda_{i}$ for every type of the fixed point (see Tables 1-3) provides the following conclusions.

1) For the three-dimensional Ising model $(p=1)$, the second-type fixed point is stable (Table 1(a)). The complex values $\lambda_{1}$ and $\lambda_{2}$ for positive $\left|\lambda_{1}\right|,\left|\lambda_{2}\right|$ and $\lambda_{3}$, show that the second-type fixed point, in contrast to the third-type one, is a stable focus in the parametric space $\left(\tilde{g}, g_{0}, g_{1}\right)$, and the renormalization group flows approach the secondtype fixed point by a spiral curve. At the threshold dimension $d_{c}=3.986$ (see Table $1(\mathrm{~b})$, (c)), the second-type fixed point looses stability ( $\lambda_{3}$ changes sign). Since all other fixed points remain unstable in the entire range of the dimension variation $3 \leq d<4$, no critical behavior is realized in the system at $3.986 \leq d$ due to the replica symmetry breaking. The analysis of the behavior of renormalization group flows at $3.986 \leq d$ provides the following results.

2) For the three-dimensional XY model $(p=2)$, small values of $\lambda_{i}$ (see Table $2(\mathrm{a})$ ) indicate that the second- type replica symmetric fixed point is weakly stable. However, already for the dimension $d_{c}=3.1$ (see Table 2(b), (c)), the third-type fixed point with RSB effects becomes stable. However, the critical behavior determined by this point is nonuniversal and depends on the parameter $x_{0}$ and, therefore, on the concentration of impurities. A stability analysis of the third-type fixed point reveals that it is stable only in the interval $0 \leq x_{0} \leq x_{c}(d)$, where $x_{c}$ is a threshold value of the parameter, which depends on the dimension of the system. For example, for $d=3.1 x_{c}=0.1$; and, for $d=3.999 x_{c}=0.3$ In the interval $x_{c}(d)<x_{0}<1$, all fixed points are unstable.

3) For the isotropic three-dimensional Heisenberg model $(p=3)$, the first-type fixed point becomes stable (Table $3(\mathrm{a})$ ), while at the other fixed points the constants $g_{0}^{*}$ and $g_{1}^{*}$ take physically senseless negative values. Only at $d_{c}=3.999, g_{0}^{*}$ and $g_{1}^{*}$ take physically meaningful values for the third-type point, and this point becomes stable in the interval $0 \leq x_{0} \leq 0.4$ (Table 3(b), (c)). In the interval $0.4<x_{0}<1$, all fixed points are unstable. 
Table 1

Coordinates of the FPs and eigenvalues of the stability matrix for $p=1$ :

a) dimension $d=3.0$

\begin{tabular}{|c|c|c|c|c|cc|c|}
\hline Type & $x_{0}$ & $\tilde{g}^{*}$ & $g_{0}^{*}$ & $g_{1}^{*}$ & $\lambda_{1}$ & $\lambda_{2}$ & $\lambda_{3}$ \\
\hline 1 & & 0.1774 & 0 & 0 & 0.6536 & -0.1692 & -0.1692 \\
\hline 2 & & 0.1844 & 0.0812 & 0.0812 & $0.5253 \pm 0.0893 i$ & 0.2112 \\
\hline 3 & 0.0 & 0.1844 & 0 & 0.0812 & $0.5253 \pm 0.0893 i$ & -0.0392 \\
& 0.1 & 0.1840 & 0 & 0.0829 & $0.5352 \pm 0.0983 i$ & -0.0492 \\
& 0.2 & 0.1835 & 0 & 0.0846 & $0.5471 \pm 0.1067 i$ & -0.0599 \\
& 0.3 & 0.1830 & 0 & 0.0863 & $0.5607 \pm 0.1133 i$ & -0.0712 \\
& 0.4 & 0.1824 & 0 & 0.0880 & $0.5765 \pm 0.1180 i$ & -0.0832 \\
& 0.5 & 0.1817 & 0 & 0.0895 & $0.5951 \pm 0.1203 i$ & -0.0959 \\
& 0.6 & 0.1810 & 0 & 0.0910 & $0.6172 \pm 0.1189 i$ & -0.1093 \\
& 0.7 & 0.1802 & 0 & 0.0924 & $0.6439 \pm 0.1114 i$ & -0.1234 \\
& 0.8 & 0.1793 & 0 & 0.0936 & $0.6760 \pm 0.0921 i$ & -0.1381 \\
& 0.9 & 0.1784 & 0 & 0.0947 & $0.7135 \pm 0.0353 i$ & -0.1534 \\
& 1.0 & 0.1774 & 0 & 0.0957 & 0.8573 & 0.6536 & -0.1692 \\
\hline
\end{tabular}

b) dimension $d=3.985$

\begin{tabular}{|c|c|c|c|c|cc|c|}
\hline Type & $x_{0}$ & $\tilde{g}^{*}$ & $g_{0}^{*}$ & $g_{1}^{*}$ & $\lambda_{1}$ & $\lambda_{2}$ & $\lambda_{3}$ \\
\hline 1 & & 0.0917 & 0 & 0 & 0.6315 & -0.4163 & -0.4163 \\
\hline 2 & & 0.1231 & 0.1090 & 0.1090 & $0.6986 \pm 0.1311 i$ & 0.0022 \\
\hline 3 & 0.0 & 0.1231 & 0 & 0.1090 & $0.7047 \pm 0.1069 i$ & -0.0363 \\
\hline
\end{tabular}

c) dimension $d=3.986$

\begin{tabular}{|c|c|c|c|c|cc|c|}
\hline Type & $x_{0}$ & $\tilde{g}^{*}$ & $g_{0}^{*}$ & $g_{1}^{*}$ & $\lambda_{1}$ & $\lambda_{2}$ & $\lambda_{3}$ \\
\hline 1 & & 0.0916 & 0 & 0 & 0.6318 & -0.4165 & -0.4165 \\
\hline 2 & & 0.1230 & 0.1092 & 0.1092 & $0.6895 \pm 0.1453 i$ & -0.0076 \\
\hline 3 & 0.0 & 0.1230 & 0 & 0.1092 & $0.7018 \pm 0.0935 i$ & -0.0359 \\
\hline
\end{tabular}


Table 2

Coordinates of the FPs and eigenvalues of the stability matrix for $p=2$ :

a) dimension $d=3.0$

\begin{tabular}{|c|c|c|c|c|c|c|c|}
\hline Type & $x_{0}$ & $\tilde{g}^{*}$ & $g_{0}^{*}$ & $g_{1}^{*}$ & $\lambda_{1}$ & $\lambda_{2}$ & $\lambda_{3}$ \\
\hline 1 & & 0.155830 & 0 & 0 & 0.667315 & -0.001672 & -0.001672 \\
\hline 2 & & 0.155831 & 0.000584 & 0.000584 & 0.667312 & 0.001682 & 0.000004 \\
\hline 3 & 0.0 & 0.155831 & 0 & 0.000584 & 0.667313 & 0.001683 & -0.000001 \\
& 0.1 & 0.155831 & 0 & 0.000614 & 0.667313 & 0.001684 & -0.000088 \\
& 0.2 & 0.155831 & 0 & 0.000648 & 0.667313 & 0.001685 & -0.000186 \\
& 0.3 & 0.155831 & 0 & 0.000686 & 0.667313 & 0.001686 & -0.000296 \\
& 0.4 & 0.155831 & 0 & 0.000729 & 0.667313 & 0.001687 & -0.000419 \\
& 0.5 & 0.155831 & 0 & 0.000778 & 0.667313 & 0.001687 & -0.000559 \\
& 0.6 & 0.155831 & 0 & 0.000833 & 0.667313 & 0.001688 & -0.000717 \\
& 0.7 & 0.155831 & 0 & 0.000896 & 0.667314 & 0.001690 & -0.000901 \\
& 0.8 & 0.155831 & 0 & 0.000971 & 0.667314 & 0.001692 & -0.001116 \\
& 0.9 & 0.155831 & 0 & 0.001058 & 0.667315 & 0.001694 & -0.001369 \\
& 1.0 & 0.155830 & 0 & 0.001163 & 0.667316 & 0.001696 & -0.001672 \\
\hline
\end{tabular}

b) dimension $d=3.10$

\begin{tabular}{|c|c|c|c|c|c|c|c|}
\hline Type & $x_{0}$ & $\tilde{g}^{*}$ & $g_{0}^{*}$ & $g_{1}^{*}$ & $\lambda_{1}$ & $\lambda_{2}$ & $\lambda_{3}$ \\
\hline 1 & & 0.1499955 & 0 & 0 & 0.689608 & -0.009539 & -0.009539 \\
\hline 2 & & 0.1500170 & 0.00325 & 0.00325 & 0.689535 & 0.009887 & -0.000003 \\
\hline 3 & 0.0 & 0.1500170 & 0 & 0.00325 & 0.689535 & 0.009887 & 0.000109 \\
& 0.1 & 0.1500169 & 0 & 0.00341 & 0.689535 & 0.009899 & -0.000401 \\
& 0.2 & 0.1500167 & 0 & 0.00360 & 0.689536 & 0.009926 & -0.000961 \\
\hline
\end{tabular}

c) dimension $d=3.999$

\begin{tabular}{|c|c|c|c|c|c|c|c|}
\hline Type & $x_{0}$ & $\tilde{g}^{*}$ & $g_{0}^{*}$ & $g_{1}^{*}$ & $\lambda_{1}$ & $\lambda_{2}$ & $\lambda_{3}$ \\
\hline 1 & & 0.089762 & 0 & 0 & 1.119442 & -0.133591 & -0.133591 \\
\hline 2 & & 0.092307 & 0.036991 & 0.036991 & 1.103421 & 0.227335 & -0.025378 \\
\hline 3 & 0.0 & 0.092307 & 0 & 0.036991 & 1.103421 & 0.227335 & 0.030783 \\
& 0.1 & 0.092270 & 0 & 0.038723 & 1.102142 & 0.235506 & 0.021563 \\
& 0.2 & 0.092205 & 0 & 0.040559 & 1.100913 & 0.244667 & 0.011135 \\
& 0.3 & 0.092108 & 0 & 0.042500 & 1.099845 & 0.254810 & -0.000648 \\
& 0.4 & 0.091970 & 0 & 0.044547 & 1.099106 & 0.265820 & -0.013939 \\
\hline
\end{tabular}


Table 3

Coordinates of the FPs and eigenvalues of the stability matrix for $p=3$ :

a) dimension $d=3.0$

\begin{tabular}{|c|c|c|c|c|c|c|c|}
\hline Type & $x_{0}$ & $\tilde{g}^{*}$ & $g_{0}^{*}$ & $g_{1}^{*}$ & $\lambda_{1}$ & $\lambda_{2}$ & $\lambda_{3}$ \\
\hline 1 & & 0.1383 & 0 & 0 & 0.6814 & 0.1315 & 0.1315 \\
\hline 2 & & 0.1419 & -0.0359 & -0.0359 & 0.6727 & -0.0891 & -0.1450 \\
\hline 3 & 0.0 & 0.1419 & 0 & -0.0359 & 0.6727 & -0.0891 & -0.0058 \\
& 0.1 & 0.1420 & 0 & -0.0382 & 0.6727 & -0.0865 & 0.0011 \\
& 0.2 & 0.1420 & 0 & -0.0408 & 0.6728 & -0.0836 & 0.0088 \\
& 0.3 & 0.1421 & 0 & -0.0439 & 0.6730 & -0.0802 & 0.0175 \\
& 0.4 & 0.1420 & 0 & -0.0474 & 0.6734 & -0.0764 & 0.0273 \\
& 0.5 & 0.1420 & 0 & -0.0516 & 0.6738 & -0.0719 & 0.0385 \\
& 0.6 & 0.1418 & 0 & -0.0565 & 0.6745 & -0.0668 & 0.0515 \\
& 0.7 & 0.1415 & 0 & -0.0625 & 0.6755 & -0.0606 & 0.0667 \\
& 0.8 & 0.1409 & 0 & -0.0699 & 0.6768 & -0.0533 & 0.0845 \\
& 0.9 & 0.1400 & 0 & -0.0793 & 0.6787 & -0.0443 & 0.1058 \\
& 1.0 & 0.1383 & 0 & -0.0915 & 0.6814 & -0.0331 & 0.1315 \\
\hline
\end{tabular}

b) dimension $d=3.999$

\begin{tabular}{|c|c|c|c|c|c|c|c|}
\hline Type & $x_{0}$ & $\tilde{g}^{*}$ & $g_{0}^{*}$ & $g_{1}^{*}$ & $\lambda_{1}$ & $\lambda_{2}$ & $\lambda_{3}$ \\
\hline 1 & & 0.081989 & 0 & 0 & 1.113633 & -0.000820 & -0.000820 \\
\hline 2 & & 0.081989 & 0.000171 & 0.000171 & 1.113633 & 0.000822 & -0.000228 \\
\hline 3 & 0.0 & 0.081989 & 0 & 0.000171 & 1.113633 & 0.000822 & 0.000228 \\
& 0.1 & 0.081989 & 0 & 0.000183 & 1.113633 & 0.000822 & 0.000188 \\
& 0.2 & 0.081989 & 0 & 0.000196 & 1.113633 & 0.000823 & 0.000142 \\
& 0.3 & 0.081989 & 0 & 0.000212 & 1.113633 & 0.000823 & 0.000088 \\
& 0.4 & 0.081989 & 0 & 0.000230 & 1.113633 & 0.000823 & 0.000025 \\
& 0.5 & 0.081989 & 0 & 0.000251 & 1.113633 & 0.000823 & -0.000050 \\
& 0.6 & 0.081989 & 0 & 0.000277 & 1.113633 & 0.000824 & -0.000140 \\
& 0.7 & 0.081989 & 0 & 0.000309 & 1.113633 & 0.000824 & -0.000251 \\
& 0.8 & 0.081989 & 0 & 0.000350 & 1.113633 & 0.000825 & -0.000391 \\
& 0.9 & 0.081989 & 0 & 0.000402 & 1.113633 & 0.000826 & -0.000574 \\
& 1.0 & 0.081989 & 0 & 0.000473 & 1.113633 & 0.000828 & -0.000820 \\
\hline
\end{tabular}


Critical exponents of the 3D models for RS FP's

\begin{tabular}{|lllccccc|}
\hline Model & FP & & $\eta$ & $\nu$ & $\gamma$ & $\beta$ & $\alpha$ \\
\hline Ising & RS1 & this work & 0.028 & 0.631 & 1.244 & 0.324 & 0.107 \\
& & Ref. [16] & $0.031(4)$ & $0.630(2)$ & $1.241(2)$ & $0.325(2)$ & $0.110(5)$ \\
& RS2 & this work & 0.028 & 0.672 & 1.329 & 0.345 & -0.015 \\
& & Ref. [17] & $0.030(3)$ & $0.678(10)$ & $1.330(17)$ & $0.349(5)$ & $-0.034(30)$ \\
\hline XY & \multirow{2}{*}{ RS1 } & this work & 0.029 & 0.667 & 1.318 & 0.343 & -0.001 \\
& & Ref. [16] & $0.034(3)$ & $0.669(1)$ & $1.316(1)$ & $0.346(1)$ & $-0.007(6)$ \\
\hline Heisenberg & RS1 & this work & 0.028 & 0.697 & 1.379 & 0.369 & -0.092 \\
& & Ref. [16] & $0.034(3)$ & $0.705(1)$ & $1.387(1)$ & $0.364(1)$ & $-0.115(9)$ \\
\hline
\end{tabular}

Note that although the calculations indicate the stability of the impurity replica symmetric second-type fixed point, there is reason to believe that, in the higher order approximations (as is the case for disordered systems considered without taking into account RSB effects [1] ), the first-type fixed point, which corresponds to the critical behavior of the homogeneous system, will become stable. On the one hand, this is indicated by the very weak stability $\left(\lambda_{3}=0.000004\right)$ of the second-type fixed point and by the fact that the threshold value of the order parameter $p_{c}=2.0114$ found in the two-loop approximation, which separates the critical behavior domains determined by the first- $\left(p>p_{c}\right)$ and second-type $\left(p<p_{c}\right)$ fixed points, is very close to $p=2$. This explains a very slow variation of the eigenvalues $\lambda_{i}$ of the stability matrix for the disordered XY model with the variation of the system dimension (Table 2). On the other hand, the negative value of the critical heat capacity coefficient $\alpha$ of the XY model also suggests, according to the Harris criterion, that the critical behavior of the model is stable with respect to the influence of the quenched disorder and, therefore, it can be expected that $p_{c}<2$ in the higher order approximations. For example, the value $p_{c}=1.912(4)$ was found in [15] on the basis of the six-loop approximation with the use of the pseudo $\varepsilon$-expansion and the Pade Borel Leroy summation technique with a thoroughly chosen fitting parameter.

Because $p_{c}$ is very close to $p=2$ for the XY model, one can expect that the calculations based on higher order approximations will substantially change the threshold dimension $d_{c}(p=2)$, although for the Ising and Heisenberg models, the changes of $d_{c}(p)$ should 
be small. This assumption is supported by the calculation of critical indexes for threedimensional homogeneous models with $p=1,2,3$ and the disordered Ising model. We performed these calculations in the two-loop approximation with the use of the Pade Borel summation technique (Table 4). The comparison of these results with the corresponding indexes reported in [16, 17, where the all-time accurate calculations for threedimensional models were performed in the six-loop approximation, shows that the difference in the values of critical indexes does not exceed 0.02 .

The values of the threshold dimensions $d_{c}(p)$, which separate the domain of critical behavior with RSB effects $d_{c}(p)<d<4$ from the domain where these effects are inessential, can be considered as threshold dimensions that restrict the scope of the $\varepsilon$-expansion method as applied to the three-vertex model of the weakly disordered system and the corresponding results reported in [4, 5, 6]. Our analysis also shows that the results of application of the $\varepsilon$-expansion technique to multivertex statistical models are unreliable independently of the approximation order. This is explained by the competition between different types of fixed points in the parametric space of multivertex models, which usually does not allow one to pass to the limit as $\varepsilon \rightarrow 1$ without crossing the marginal dimensions $3 \leq d_{c}<4$ separating the stability domains of different fixed points.

To reveal the character of the behavior of a disordered system with RSB effects in the domain without stable critical states, we analyzed the phase portrait of the model based on the system of equations

$$
r \frac{\partial g_{i}}{\partial r}=\beta_{i}\left(\tilde{g}, g_{0}, g_{1}\right)
$$

which specifies phase trajectories in the space of vertices $\left(\tilde{g}, g_{0}, g_{1}\right)$. An analysis shows (see Fig. 2) that, for the Ising model with $d_{c}=3.986$ at $d \geq 3.986$, where none of the fixed points is stable, the strong coupling regime with the renormalization group flows determined by $\left(\tilde{g}, g_{0}, g_{1}\right) \rightarrow(\infty, 0,0)$ is realized if $\tilde{g}>\tilde{g}^{*}$. At the same time, at $\tilde{g}<\tilde{g}^{*}$, the flows with $\left(\tilde{g}, g_{0}, g_{1}\right) \rightarrow(0,0,0)$ are realized, which asymptotically approach the Gauss fixed point $(0,0,0)$ and then also tend to infinity along the axes $\tilde{g}, g_{0}, g_{1}$. Such a behavior of the flows at $\tilde{g}<\tilde{g}^{*}$ is caused by the closeness of the system dimension $d$ to four when the effect of fluctuations is negligibly small and the Gauss fixed point becomes an attractor. 


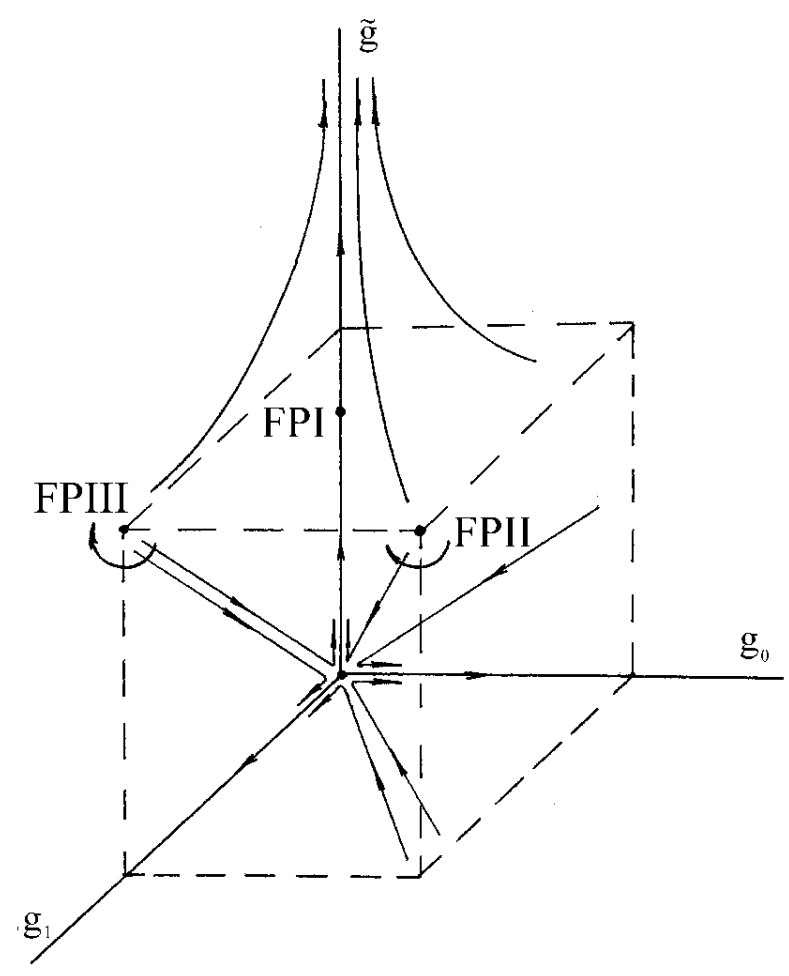

Figure 2: The picture of renormalization group flows in the parametric space $\left(\tilde{g}, g_{0}, g_{1}\right)$ for the Ising model with the system dimension $\mathrm{d}=3.99$.

\section{Conclusions}

The renormalization group analysis of weakly disordered systems of an arbitrary dimension in the range from three to four conducted in the two-loop approximation showed that the critical behavior of threedimensional systems is stable with respect to the effect of the replica symmetry breaking. In systems with a one-component order parameter, the critical behavior determined by the structural disorder with a replica symmetric fixed point is realized. The presence of weak disorder does not affect the critical behavior of multicomponent systems, although the proof of this fact for systems with $p=2$ requires calculations with higher order approximations.

Effects of the replica symmetry breaking manifest themselves only in disordered systems with the dimension greater than three, and the threshold dimensions $d_{c}$ depend on the number of components of the order parameter $p$ and the value of the parameter $x_{0}$. The predicted picture of the influence of replica symmetry breaking on the critical behavior of disordered systems with a dimension $d>d_{c}$ qualitatively agrees with the results reported in 4, 5, 6]. The latter results were obtained on the basis of the $\varepsilon$-expansion 
technique. For systems with $p=1$, RSB effects destroy the stable critical behavior, and the strong coupling regime is realized; for systems with $p=2$ and 3 , a domain of nonuniversal critical behavior occurs at $0 \leq x_{0} \leq x_{c}(d)$. For $x_{0}$ outside this interval, the system exhibits no critical behavior, as is the case at $p=1$.

The values of threshold dimensions $d_{c}(p): d_{c}(p=1)=3.986, d_{c}(p=2)=3.10$, and $d_{c}(p=3)=3.999$ which separate the domain of critical behavior with RSB effects $\left.d_{c}(p)<d<4\right)$ from the domain where these effects are insignificant, simultaneously specify the lower bound of the domain where the results obtained by $\varepsilon$-expansion are applicable to the description of the model of weakly disordered systems with RSB effects [4, 5, 6]. It is noted that calculations carried out in higher order approximations of the theory can significantly change the threshold dimension dc for the XY model. On the other hand, changes in $d_{c}(p)$ for the Ising and Heisenberg models are expected to be small, which leaves the scope of the results obtained by the $\varepsilon$-expansion technique close to dimension four.

As the concentration of defects increases, one can expect a decrease of the threshold values $d_{c}$ down to $d_{c}<3$ beginning with a certain threshold concentration. In this case, the influence of replica symmetry breaking effects can be significant. Due to specific features of the manifestation of RSB effects, the concentration ns corresponding to the spin percolation threshold can play the role of the threshold concentration of defects for the Ising model, so that no stable critical behavior is observed at $n>n_{s}$. For the $\mathrm{XY}$ and Heisenberg models, this role can be played by the concentration of defects corresponding to the impurity percolation threshold $n_{\text {imp }}=1-n_{s}$ with a nonuniversal critical behavior for $n_{\text {imp }}<n<n_{s}$ and the absence of a stable critical behavior at $n>n_{s}$.

\section{Acknowledgmets}

This work was supported in part by the Ministry of Education of the Russian Federation through Grants No. UR.01.01.052 and No. E02-3.2-196. 
[1] S.F. Edwards, P.W. Anderson. J.Phys. F5, 965 (1975).

[2] J. Emery. Phys. Rev. B11, 239 (1975).

[3] G. Grinstein, A. Luther. Phys. Rev. B13, 1329 (1976).

[4] Vik.S.Dotsenko, A.B.Harris, D.Sherrington, R.B.Stinchcombe. J.Phys.A28, 3093 (1995).

[5] Vik.S. Dotsenko, D.E. Feldman. J.Phys. A28, 5183 (1995).

[6] Vik. S. Dotsenko, Uspekhi Fizicheskikh Nauk, 165, 481 (1995).

[7] V. V. Prudnikov, A. V. Ivanov, A. A. Fedorenko, Sov. Phys. JETP Lett. 66835 (1997); V. V. Prudnikov, S. V. Belim, A. V. Ivanov, E. V. Osintsev, A. A. Fedorenko, Sov. Phys. JETP 87527 (1998); V. V. Prudnikov, P. V. Prudnikov, A. A. Fedorenko, Sov. Phys. JETP Lett. 68950 (1998); Phys.Rev. B 62, 8777 (2000).

[8] S. A. Antonenko, A. I. Sokolov, Phys.Rev. B 49, 15901 (1994); A.I. Sokolov, K.B. Varnashev, A.I. Mudrov. Int. J. Mod. Phys. B 12, 1365 (1998); A.I. Sokolov, K.B. Varnashev, Phys.Rev. B 59, 8363 (1999).

[9] V. V. Prudnikov, P. V. Prudnikov, A. A. Fedorenko, Sov. Phys. JETP Lett. 73,153 (2001).

[10] V.V. Prudnikov, P.V. Prudnikov, A.A. Fedorenko. Phys.Rev. B63, 184201 (2001).

[11] A. Pelissetto, E. Vicari. Phys.Rev. B62, 6393 (2000).

[12] V. V. Prudnikov, A. N. Vakilov, Sov. Phys. JETP 103, 962 (1993).

[13] G. Parisi. J.Phys. A13, 1101 (1980); G. Parisi. J.Phys. A13, L115 (1980); G. Parisi. J.Phys. A13, 1887 (1980); M. Mezard, G. Parisi, M. Virasoro. Spin-Glass Theory and Beyond, Singapore: World Scientific, 1987; Vik. S. Dotsenko, Uspekhi Fizicheskikh Nauk 163, 1 (1993).

[14] J. Zinn-Justin. Quantum Field Theory and Critical Phenomena, Clarendon, Oxford, 1996. 
[15] M. Dudka, Yu. Holovatch, T. Yavorskii. J.Phys.Stud. 5, 233 (2001).

[16] J.C. LeGuillou, J. Zinn-Justin. Phys. Rev. B21, 3976 (1980).

[17] A. Pelissetto, E. Vicari, e-print cond-mat/0002402. 